\section{Formation of cubic structures in microemulsions containing equal volumes of oil and water}

\section{J. Tabony}

CEA-IRDI-DESICP, Département de Physico-Chimie, Centre d'Études Nucléaires de Saclay, 91191 Gif-sur-Yvette Cedex, France

Microemulsions are dispersions of oil and water stabilized by an interfacial film of surfactant and co-surfactant ${ }^{1}$. Frequently a short-chain alcohol, such as butanol or pentanol, is used as the co-surfactant. Stable dispersions can often be formulated over a wide range of oil and water volume fractions. In the regions of the phase diagram containing low concentrations of either oil or water, the structure has frequently been found to be one of oil droplets in water or water droplets in oil ${ }^{2}$. In many cases by simply changing the volume fractions of oil and water, it is possible to effect a gradual transition from an oil-in-water microemulsion to a water-in-oil microemulsion. This raises the question of the structure of the microemulsion in the intermediate region where there are approximately equal volumes of oil and water, and also that of the mechanism of the structural inversion. Microemulsions of such a type are frequently thought of as being constituted by ill-defined oil and water regions arranged with an almost total absence of long-range order ${ }^{3}$. However, I now report neutron small-angle scattering measurements ${ }^{4}$ on one such microemulsion containing tetradecyl trimethylammonium bromide, butanol, octane and water which shows a well defined diffraction pattern consistent with a cubic arrangement of oil and water domains. This result shows that microemulsions of this type can be considerably more structured than presently thought and can sometimes resemble cubic liquid crystals.

Figure $1 a$ shows the neutron small-angle scattering spectrum measured from a microemulsion containing approximately equal volumes of oil and water and having the following overall composition: tetradecyl trimethylammonium bromide $(2.1 \mathrm{~g})$, butanol $(1.5 \mathrm{ml})$, octane $(4 \mathrm{ml})$ and water $(4 \mathrm{ml})$. The microemulsion is optically isotropic and of low viscosity. The spectrum shows a peak similar to that observed in other microemulsions of similar composition ${ }^{4,5}$. The radius of curvature of the spheroidal domains, as estimated from the scattering curve, is $50 \AA$. Consistent with its low viscosity, this microemulsion has little or no long-range order and individual Bragg peaks are not observed. A second system was therefore formulated whereby the extent of long-range order in the microemulsion was increased by decreasing the alcohol content. Addition of alcohol to surfactant-water lyotropic liquid crystals tends to destroy the long-range order in these mesophases. As there is a relatively large amount of alcohol (2.5 molecules of butanol for each molecule of surfactant) in the first mocroemulsion, one may expect the reverse process to occur-reducing the alcohol content could increase the long-range order in the system and lead to the appearance of resolved Bragg peaks in the neutron spectrum. This turns out to be the case. Figure $1 b$ shows the neutron small-angle spectrum from a dispersion containing $30 \%$ less alcohol than before $(1 \mathrm{ml}$ of butanol instead of $1.5 \mathrm{ml})$. The microemulsion is now extremely viscous but remains optically isotropic. The broad envelope seen in Fig. $1 a$ has split and at least five diffraction peaks are clearly resolved. The position of the most intense peak remains unchanged and this suggests that, apart from the extent of long-range order, the structure of the microemulsion is unaffected by reducing the alcohol content. The diffraction pattern shown in Fig. $1 b$ indexes onto a cubic lattice. In cubic liquid crystals it is difficult to distinguish between primitive, face-centred, body-centred and diamond-like structures; however, the observed spectrum seems to be in better agreement with a diamond arrangement than with the others.
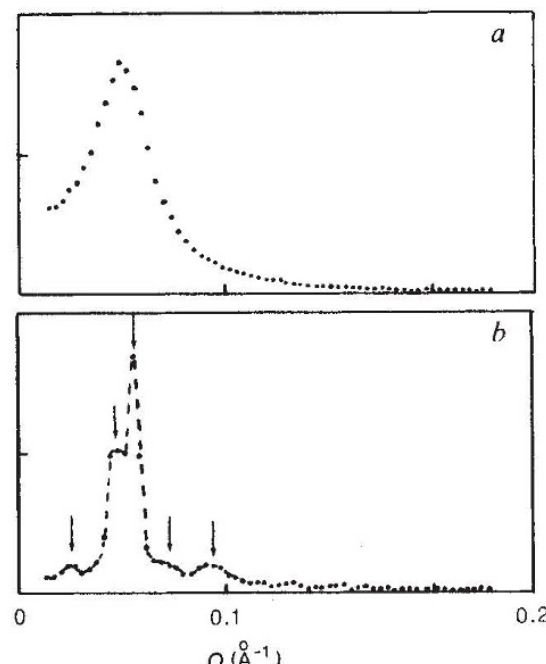

Fig. 1 Neutron small-angle scattering spectra from microemulsions containing equal volumes of oil and water (tetradecyl trimethylammonium bromide, $2.1 \mathrm{~g}$; octane, $4 \mathrm{ml}$; and water, $4 \mathrm{ml}$ ) but having different butanol contents: $a, 1.5 \mathrm{ml} ; b, 1.0 \mathrm{ml}$. The spectrum shown in $b$ indexes onto a cubic structure. The spectra were measured using the D16 camera ${ }^{10}$ at the Institut LaueLangevin, Grenoble on samples containing $\mathrm{D}_{2} \mathrm{O}$ as the only deuterated component.

In the phase diagram, the viscous isotropic phase borders onto a birefringent phase which may have a lamellar structure, while the fluid phase of higher alcohol content meets up at lower surfactant concentrations with microemulsions which show Winsor I-Winsor III-Winson II phase transitions with added $\mathrm{sal}^{t^{6}}$. A similar phase behaviour occurs for the SDS/butanol/ toluene/water system. The viscous isotropic phase obtained in this system also shows a diffraction pattern similar to that shown in Fig. $1 b$, and such cubic structures may well be a general feature of this type of microemulsion.

Of the different possible cubic structures, the most likely appears to be the interwoven bicontinuous arrangement proposed by Scriven ${ }^{7}$. However, the possibility that the microemulsion is comprised of an organized dispersion of oil or water or droplets cannot, for the time being, be discounted. The latter type of structure would support the interpretation of Kotlarchyk et $\mathrm{al}^{5}$, whose results were obtained using a different type of surfactant.

The observation of cubic diffraction patterns from a microemulsion containing equal volumes of oil and water, shows not only that the oil and water domains are arranged on a cubic lattice, but also that under some circumstances considerable long-range order may exist. Rather than having ill-defined structures, some of these microemulsions show a strong resemblance to the cubic liquid crystalline mesophases ${ }^{8,9}$ that occur in surfactant-water binary mixtures.

I thank the Institut Laue-Langevin for providing neutron beam time, and S. Wilson and L. Braganza for assistance with the D16 diffractometer.

Received 12 July; accepted 8 October 1985.

1. Prince, L. M. Microemulsions. Theory and Practice (Academic, New York, 1977)

2. Cebula, D. J., Harding, L., Ottewill, R. H. \& Pusey, P. N. Colloid Polym. Sci. 258, 973-97x (1980).

3. Talmon, Y. \& Prager, S. J. chem. Phys. 69, 2984 (1978).

4. de Geyer, A. \& Tabony, J. Chem. Phys. Lett. 113, 83 (1985)

5. Kotlarchyk, M., Chen, S. H., Huang, J. S. \& Kim, M. W. Phys. Rev. Lett. 53, 941 (1984).

6. Winsor, P. A. Solvent Properties of Amphiphilic Compounds (Butterworths, London, 1954)

7. Scriven, L. E. Nature 263, 123 (1976).

8. Elkwall, P. in Advances in Liquid Crystals (ed. Brown, G. H.) Vol. 1, Ch. 1 (Academic, New York, 1971).

9. Tiddy, G. J. T. Phys. Rep. 59 (1980)

10. Neutron Beam Facilities Available for Users (Institut Laue-Langevin, Grenobles, 1981). 